\title{
Preparation of Nickel-Cobalt/Carborundum Carbide Composite Coatings by Supergravity Field-Enhanced Electrodeposition
}

Xiaoyun $\mathrm{Hu}^{1 *}$ and Ningsong Qu,

\begin{abstract}
Nickel-cobalt/silicon carbide (Ni-Co/SiC) composite coatings were fabricated by supergravity field-enhanced electrodeposition. The surface morphology and the distribution of the SiC particles in the coatings were examined by scanning electron microscope and energy dispersive $X$-ray spectrometry. The preferred orientations of the coatings were measured by $X$-ray diffractometry. The wear resistance and microhardness were measured by a reciprocating tribometer and a microhardness instrument, respectively. The results revealed that the use of the supergravity field enhanced the smoothness of the as-deposited Ni-Co/SiC coatings, and the SiC nanoparticles were uniformly distributed in comparison with that for conventional electrodeposition. When the rotation speed of the cathode, which provided the supergravity field, was $800 \mathrm{r} / \mathrm{min}$, the $\mathrm{SiC}$ content in the coating reached a maximum of $8.1 \mathrm{wt} \%$, which was a much higher content than the $2.2 \mathrm{wt} \%$ value obtained under conventional electrodeposition. The highest coating microhardness of $680 \mathrm{HV}$ was also observed at this rotation speed. In addition, the wear resistance of the as-prepared $\mathrm{Ni}-\mathrm{Co} / \mathrm{SiC}$ coatings exhibited improved performance relative to that prepared under normal gravity. A minimum wear weight loss of $1.4 \mathrm{mg}$ together with an average friction coefficient of 0.13 were also realized at a rotation speed of 800 $\mathrm{r} / \mathrm{min}$, values which were much lower than those for normal gravity.
\end{abstract}

Keywords: Supergravity field, Electrodeposition, Microhardness, Wear resistance

\section{Introduction}

Nickel-cobalt (Ni-Co) alloy coatings as an important engineering material exhibit many attractive features, such as high hardness, good wear and corrosion resistance, and good magnetoconductivity, thermal conductivity and electrocatalysis activity [1-4]. The coatings have been used extensively in diverse fields including magnetic sensor technology [5], aerospace, micro-electromechanical systems and nano-electromechanical systems [6]. Nowadays, the consensus is that particle-reinforced composite coatings exhibit better performance than alloy coatings [7-9]. Thus, to further improve certain properties of the target components, insoluble particles

\footnotetext{
*Correspondence: hxyun@nuaa.edu.cn

${ }^{1}$ Nanjing University of Aeronautics and Astronautics, Nanjing 210016,

China

Full list of author information is available at the end of the article
}

are usually incorporated into the alloy coatings. Given the attractive features of $\mathrm{Ni}$-Co alloys, much work has been done concerning the Ni-Co matrix. For instance, $\mathrm{Cr}_{2} \mathrm{O}_{3}$ [10], $\mathrm{Al}_{2} \mathrm{O}_{3}[11,12]$ and diamond [13] have been successfully embedded into the $\mathrm{Ni}$-Co matrix such that enhanced properties for these composite coatings have been obtained.

Among the particulates used for strengthening purposes, $\mathrm{SiC}$ possesses excellent chemical stability and mechanical characteristics in severe environments and has been widely investigated [14-17]. Given the importance of $\mathrm{Ni}$-Co coatings, much attention has been directed at $\mathrm{Ni}-\mathrm{Co} / \mathrm{SiC}$ composite coatings, and many studies have focused on electrodeposition whereby insoluble particles with metallic cations, which are suspended in electrolyte, undergo co-deposition [18]. Bakhit suggested that $\mathrm{Ni}-\mathrm{Co} / \mathrm{SiC}$ coatings, which are reinforced by the nanosized $\mathrm{SiC}$ particles, possess enhanced 
properties in comparison with those of micro-sized $\mathrm{SiC}$ particles [18]. However, in the case of nanoscale particles, researchers have reported that it is more difficult to incorporate nanosized particles than micro-sized ones due to agglomeration [19], which may lead to a drastic decrease in the mechanical properties of the composite coatings [20] and reduce the co-deposition content of the particles [19]. To obtain $\mathrm{Ni}-\mathrm{Co} / \mathrm{SiC}$ composite coatings with well-dispersed nanoscale $\mathrm{SiC}$ particulates and to increase the content of $\mathrm{SiC}$ in the coatings, sodium dodecyl sulphate as surfactant has been added to the electrolyte solution [7]. In addition, a sediment co-deposition technique which exploits gravity was proposed to promote nanoparticle embedment [18].

Recently, the supergravity field effect has been attracting attention in electrochemistry. It has been reported that convection is enhanced and thus mass transfer is improved under the supergravity field [21, 22]. Moreover, coatings offering improved performance were achieved using electrodeposition with a supergravity field [23]. Using a supergravity field with electrodeposition, Murotani et al. increased the volume fraction of $\mathrm{SiC}$ in a $\mathrm{Ni}$ matrix and found that the morphological structure of the as-deposited film was also influenced by the supergravity field [24]. As for the $\mathrm{Ni}-\mathrm{Co} / \mathrm{SiC}$ nanocomposite coatings, although much work has been done, few studies have reported on the preparation of $\mathrm{Ni}-\mathrm{Co} / \mathrm{SiC}$ nanocomposite coatings using a supergravity field. Considering the benefits of a supergravity field, the present work concerns the fabrication of $\mathrm{Ni}-\mathrm{Co} / \mathrm{SiC}$ nanocomposite coatings. The effects of the supergravity field on the $\mathrm{SiC}$ content, the morphology, the grain size, the microhardness and the wear resistance have been investigated.

\section{Experimental Details}

The setup used here has been detailed described in previous work [25]. Various supergravity fields were generated by changing the rotation speed of the motor [21]. The anode was a hollow cylinder made of pure $\mathrm{Ni}$ with the following dimensions: thickness $2 \mathrm{~mm}$, length $35 \mathrm{~mm}$ and outer diameter $(\Phi) 40 \mathrm{~mm}$. The substrate was a hollow cylinder made of 304 SS (stainless steel) of thickness $5 \mathrm{~mm}$, length $37 \mathrm{~mm}$ and $\Phi 48$ (inner diameter). The gap between the substrate and the cathode was $4 \mathrm{~mm}$. The motor drove the cathode, positioned inside the cylindrical fixture, to rotate at user-adjustable rotation speeds. Electrolyte solution was pumped continuously into the hollow cylinder anode at constant flow. Throughout the experiment, the anode was fixed while the cathode rotated.

Based on our previous work [26], the force analysis of $\mathrm{SiC}$ particles is shown in Figure 1. The $\mathrm{SiC}$ particles suffered from five forces: gravity force $\left(G_{r a}\right)$, centrifugal force

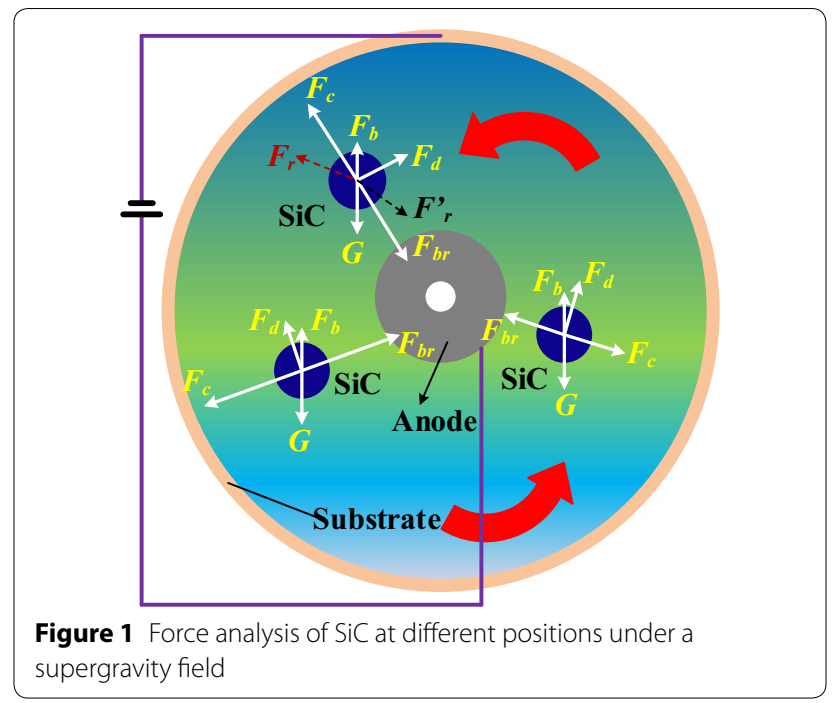

Table 1 Electrolyte composition and electrolysis
conditions

\begin{tabular}{ll}
\hline Component & Concentration \\
\hline Nickel sulphamate $(\mathrm{g} / \mathrm{L})$ & 350 \\
Nickel chloride $(\mathrm{g} / \mathrm{L})$ & 15 \\
Boric acid $(\mathrm{g} / \mathrm{L})$ & 35 \\
Cobalt sulphamate $(\mathrm{g} / \mathrm{L})$ & 40 \\
$\mathrm{SiC}(\mathrm{g} / \mathrm{L})$ & 2 \\
Electrolyte temperature $\left({ }^{\circ} \mathrm{C}\right)$ & $45 \pm 1$ \\
Current density $\left(\mathrm{A} / \mathrm{dm}^{2}\right)$ & 2 \\
Rotation speed $\left(\mathrm{r} / \mathrm{min}^{2}\right.$ & $0,400,800,1200,1600$ \\
\hline
\end{tabular}

$\left(F_{c}\right)$, buoyancy force $\left(F_{b}\right)$, drag force $\left(F_{d}\right)$, and centripetal buoyancy force $\left(F_{b r}\right)$. The drag force is generated because of the relative motion between the $\mathrm{SiC}$ particles and electrolyte. The pressure difference along the radius direction results in the centripetal buoyancy force. In this case, If the rotation speed is excessively large, the resultant force point toward the anode ( $F_{r}^{\prime}$ in Figure 1$)$, which makes the $\mathrm{SiC}$ particles travel away from the substrate. Otherwise, the resultant force directs toward the substrate $\left(F_{r}\right.$ in Figure 1), resulting in the movement of the $\mathrm{SiC}$ particles to the substrate. It is expected that the resultant force points toward the substrate, which is favorable.

On the basis of previous work [27], the chemical composition of the electrolyte solution used in this work is specified in Table 1. The average size of the $\mathrm{SiC}$ particles used was $40 \mathrm{~nm}$. Before the experiments, the electrolyte solution was stirred for $1 \mathrm{~h}$ to prevent aggregation of $\mathrm{SiC}$ and to promote dispersal of the Co ions in the electrolyte. Throughout experimentation, the thicknesses of the deposited coatings were about $60 \mu \mathrm{m}$. The rotation speed 
ranged from 0 to $1600 \mathrm{r} / \mathrm{min}$. The supergravity coefficients $G$ were obtained by Eq. (1):

$$
G=N^{2} \pi^{2} /(900 g)
$$

where $N$ denotes the rotation speed (rotations per minute); $r$ represents the radius of the fixture $(0.05 \mathrm{~m}$ in this study); and $g$ is the acceleration due to gravity $\left(9.8 \mathrm{~m} / \mathrm{s}^{2}\right)$. Under a normal gravity field, $G$ is 0 .

The surface morphology was investigated using a scanning electron microscope (SEM, S3400N Hitachi, Tokyo, Japan) with an acceleration voltage of $20 \mathrm{kV}$. The contents of $\mathrm{SiC}$ in the composite coatings were measured by an energy dispersive X-ray spectrometer linked to an electron microscope (EDS, S3400N, Hitachi, Tokyo, Japan). The measurements were performed with an operating voltage of $20 \mathrm{kV}$ a pulse count rate of $6000 \mathrm{cps}$ and a run time of $1 \mathrm{~min}$ per sample. The region examined was $100 \times 100 \mu \mathrm{m}^{2}$. For each sample, six test regions were analysed and the average of the six results was calculated. An X-ray diffractometer (XRD, X'TRA, ARL, Switzerland) with $\mathrm{CuK \alpha}$ radiation $\lambda=1.5406 \AA$ generated at $40 \mathrm{kV}$ and $40 \mathrm{~mA}$ was used to detect the preferred crystal orientation. The spectra were recorded over the $2 \theta$ range $30^{\circ}-$ $110^{\circ}$ with a scanning rate of $0.01^{\circ}$. The microhardness of the prepared $\mathrm{Ni}-\mathrm{Co} / \mathrm{SiC}$ coatings was examined using a microhardness tester (HXS-1000A, Shanghai Shangguang Instrument Plant, Shanghai, China) with a loading force of $50 \mathrm{~g}$ for $10 \mathrm{~s}$. For every as-deposited coating, the measurement was repeated five times. Wear resistance tests were carried out at ambient temperature without lubrication on a reciprocating tribometer (HEIDON, SOHGOHKEISO, Co. LTD, Japan), and the counter-body was a $\Phi 6 \mathrm{~mm}$ 304L SS ball with a hardness of HRC 63. The testing parameters were: applied load of $5 \mathrm{~N}$, frequency of $3 \mathrm{~Hz}$, stroke of $10 \mathrm{~cm}$, testing time $15 \mathrm{~min}$. The friction coefficient was recorded continuously during the test.

\section{Results and Discussion}

\subsection{Morphological Characteristics}

The SEM images of the $\mathrm{Ni}-\mathrm{Co} / \mathrm{SiC}$ composites prepared with and without a supergravity field are presented in Figure 2. It is evident that the surface morphology was rougher for the composite prepared without the supergravity field (Figure 2a) than with the supergravity field (Figure $2 \mathrm{~b}-\mathrm{e}$ ). In addition, for the former, large protrusions were present on the surface. The surface was smoother for a rotation speed of $400 \mathrm{r} / \mathrm{min}$, but spherical clusters were still observed, as shown in Figure $2 \mathrm{~b}$. When the rotation speed increased to $800 \mathrm{r} / \mathrm{min}$, the surface was further smoothened as can be seen from Figure 2c and no substantial protrusions were noted. As rotation speed further increased, the surface morphology of the coatings began to deteriorate and featured a nodular morphology. In this case, the larger the rotation speed, the rougher the surface became, as revealed by Figure $2 \mathrm{~d}$ and e. Mappings of the Si distribution for Figure 2a and c are presented in Figure $2 \mathrm{f}$ and $\mathrm{g}$. It reflects that a nonuniform distribution of $\mathrm{SiC}$ nanoparticles in the $\mathrm{Ni}-\mathrm{Co} /$ $\mathrm{SiC}$ composite coatings and agglomerations are observed in Figure 2f. However, Figure 2g indicates that a uniform distribution of the $\mathrm{SiC}$ nanoparticles in the $\mathrm{Ni}-\mathrm{Co} / \mathrm{SiC}$ composite coatings was obtained for electrodeposition with a supergravity field.

For regions in the vicinity of the peaks due to the $\mathrm{SiC}$ nanoparticles, the strength of the electric field is maximal compared with other positions [28]. As such, the cations would preferentially deposit on the coating peaks, resulting in spherical clusters being formed on the growing coating surface. However, under the action of the supergravity field, the number of peaks is reduced which leads to a flatter surface. Therefore, the supergravity field was beneficial for obtaining good surface morphology in the electrodeposition process.

\subsection{Contents of SiC and Co in the Coating}

Based on the EDS results, the contents of $\mathrm{SiC}$ in the $\mathrm{Ni}-$ $\mathrm{Co} / \mathrm{SiC}$ composite coatings under different supergravity fields are presented in Figure 3. Compared with the $\mathrm{SiC}$ content obtained in conventional electrodeposition, i.e., without a supergravity field, the contents increased remarkably when the supergravity field was applied. The maximal value of $8.1 \mathrm{wt} \%$ was achieved for a rotation speed of $800 \mathrm{r} / \mathrm{min}$. Further increases in the rotation speed resulted in a reduction in the $\mathrm{SiC}$ content. Under the supergravity field, the $\mathrm{SiC}$ nanoparticles were enriched in the vicinity of the cathode surface due to the centrifugal force, making the concentration of $\mathrm{SiC}$ higher near the diffusion layer at the cathode surface than in the bulk solution. Over a certain range of the supergravity field, the increase of rotation speed assisted in enhancing the weak $\mathrm{SiC}$ adsorption capacity [29]. On the basis of Guglielmi's two-step adsorption mechanism [30], first the $\mathrm{SiC}$ nanoparticles would have been loosely adsorbed on the cathode surface on which metal ions were deposited. Then the $\mathrm{SiC}$ particles would have been transported to the cathode surface through electrophoretic attraction and subsequently have been adsorbed on the cathode surface via Coulombic forces. With a further increase of rotation speed, the flow speed of the solution would have increased, and would have scoured the cathode surface more intensively. In this case, the $\mathrm{SiC}$ nanoparticles that were weakly adsorbed on the cathode surface were more liable to be stripped from the cathode surface. Moreover, a higher rotation speed led to a higher $\mathrm{SiC}$ concentration 

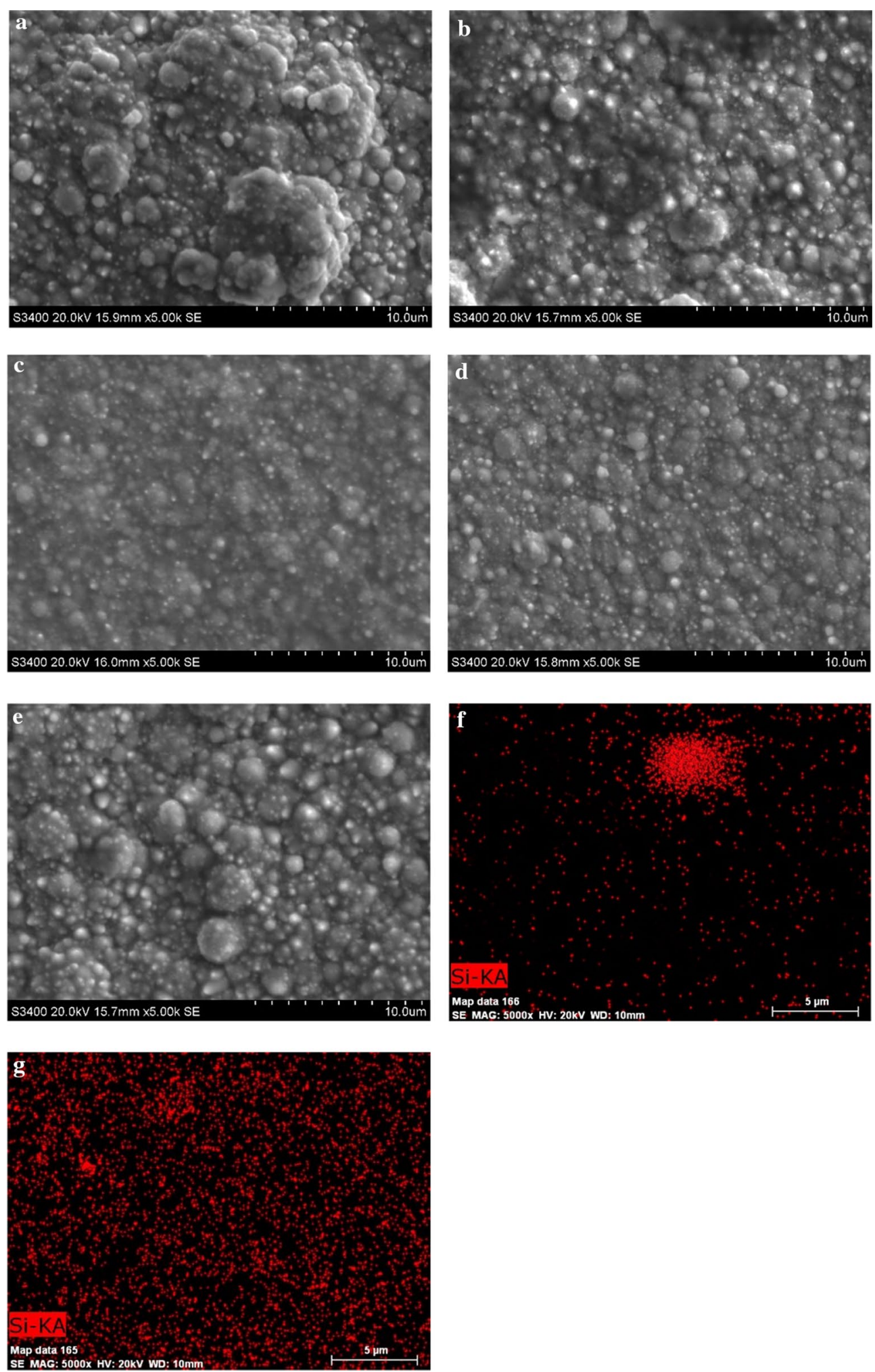

Figure 2 Morphologies of $\mathrm{Ni}-\mathrm{Co} / \mathrm{SiC}$ coatings formed at a current density of $2 \mathrm{~A} / \mathrm{dm}^{2}$ and Co content of $40 \mathrm{~g} / \mathrm{L}$ under various rotation speeds: a 0 r/min, b $400 \mathrm{r} / \mathrm{min}, \mathbf{c} 800 \mathrm{r} / \mathrm{min}$, d $1200 \mathrm{r} / \mathrm{min}$ and e $1600 \mathrm{r} / \mathrm{min}$; f Image for Si distribution of c, $\mathbf{g}$ Image for Si distribution of a 


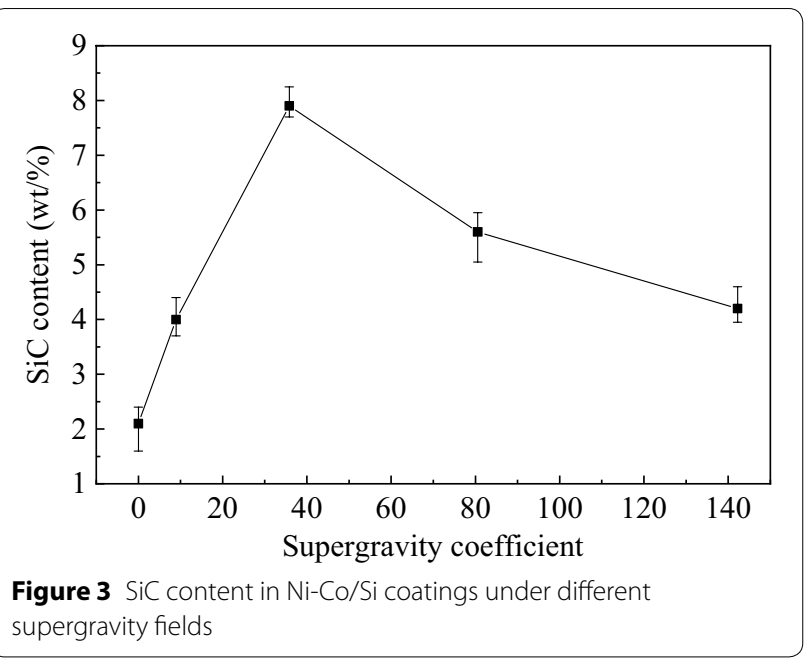

near the diffusion layer at the cathode surface, increasing the number of collisions between the nanoparticles adsorbed on the cathode surface and the ones in the bulk solution. Under such conditions, more SiC nanoparticles that were loosely adsorbed on the cathode surface would have tended to undergo desorption. Therefore, a decrease in the $\mathrm{SiC}$ content was observed at the higher rotation speeds.

\subsection{X-ray Diffraction Pattern}

The X-ray diffraction (XRD) spectra for the coatings are presented in Figure 4. It can be seen that under the normal gravity field four diffraction peaks may be observed (111), (200), (220) and (311), as shown in Figure 4a. However, when the supergravity field is introduced, the (222) diffraction peak is clearly distinct from the four above mentioned diffraction peaks, as revealed in Figure $4 \mathrm{~b}-\mathrm{e}$. In addition, all the XRD data show that the $\mathrm{Ni}-\mathrm{Co} / \mathrm{SiC}$ composite coatings are face-centred cubic structures. Peaks for $\mathrm{SiC}$ are only found in the XRD data when the rotation speeds are $800 \mathrm{r} / \mathrm{min}$ and $1200 \mathrm{r} / \mathrm{min}$ respectively. This is because the $\mathrm{SiC}$ content in the coatings prepared with rotation speeds of $800 \mathrm{r} / \mathrm{min}$ and $1200 \mathrm{r} /$ min is large enough to be detected. In other cases, the $\mathrm{SiC}$ content in the prepared coatings is too low to be detected. The texture coefficient (TC) is calculated by Eq. (2) [31] with the TC being used to evaluate the preferred crystal orientation. The TC results are illustrated in Figure 4f.

$$
T C_{h k l}=\frac{I_{h k l} / I_{h k l}^{R}}{1 / n\left(\sum I_{h k l} / I_{h k l}^{R}\right)},
$$

where $I_{h k l}$ and $I_{h k l}^{R}$ are the diffraction intensities of the crystal plane $(h k l)$ in the coating and standard samples, respectively and $n$ is the number of reflection faces in the diffraction pattern. According to Figure 4f, it is clear that under a normal gravity field, the $T C$ value of 1.4 for the plane orientation of (200) is higher than 1, which indicates the predominance of this phase. In contrast, the TC value of the plane orientation for (200) falls below 1 when rotation is applied (all conditions), suggesting its loss of predominance. Also, the $T C$ value for the plane orientation of (111) increases dramatically to a specific value much greater than 1 in each case, which demonstrates a shift of preferential orientation from (200) to (111). In comparison with the normal gravity field, the supergravity field influences the texture growth of the $\mathrm{Ni}-\mathrm{Co} / \mathrm{SiC}$ coating and the (222) phase is enhanced.

Based on the Scherrer equation given below [32], the crystallite size is calculated for the $\mathrm{Ni}-\mathrm{Co} / \mathrm{SiC}$ coatings obtained with and without the supergravity field, and the results are presented in Figure 5.

$$
F W H M=\frac{K \lambda}{D \cos \theta} \cdot \frac{180^{\circ}}{\pi},
$$

where the FWHM is the full width half maxima $(2 \theta$ degrees), $D$ is the grain size in nanometres, $K$ is a constant (generally 0.94) and the wavelength of $\mathrm{Cu} \mathrm{K \alpha}$ radiation is $0.154 \mathrm{~nm}$. It is evident that the grain size of the $\mathrm{Ni}-\mathrm{Co} / \mathrm{SiC}$ coating prepared under normal gravity is the largest, about $42.5 \mathrm{~nm}$, while the values for the samples prepared under supergravity are reduced remarkably in all instances. The minimum value of $17 \mathrm{~nm}$ is reached at a rotation speed of $800 \mathrm{r} / \mathrm{min}$. The crystallite size depends on both the nucleation rate and grain growth rate. A nucleation rate that is larger than the grain growth rate leads to a smaller grain size, whereas a dominant grain growth rate yields a larger grain size. Under the supergravity field, the $\mathrm{SiC}$ concentration in the vicinity of the diffusion layer at the cathode surface is elevated and this offers more nucleation sites [33] and subsequently leads to a smaller grain size. Moreover, $\mathrm{SiC}$ particles absorbed on cathode decrease the effective reaction area, lowering the reduction potential of matrix [34]. Therefore, nucleation rate is increased, leading to refined grain size. As discussed in the part of force analysis of $\mathrm{SiC}$ particles, when the resultant force points towards the anode, $\mathrm{SiC}$ particles move away from newly-formed coating surface. As a result, the number of $\mathrm{SiC}$ particles reduces relatively, leading to less nucleation sites and subsequently larger grain size. However, a further increase of rotation speed makes the resultant force point towards the anode. Hence, the grain size becomes relatively larger as the rotation speed increases above $1200 \mathrm{r} / \mathrm{min}$. 

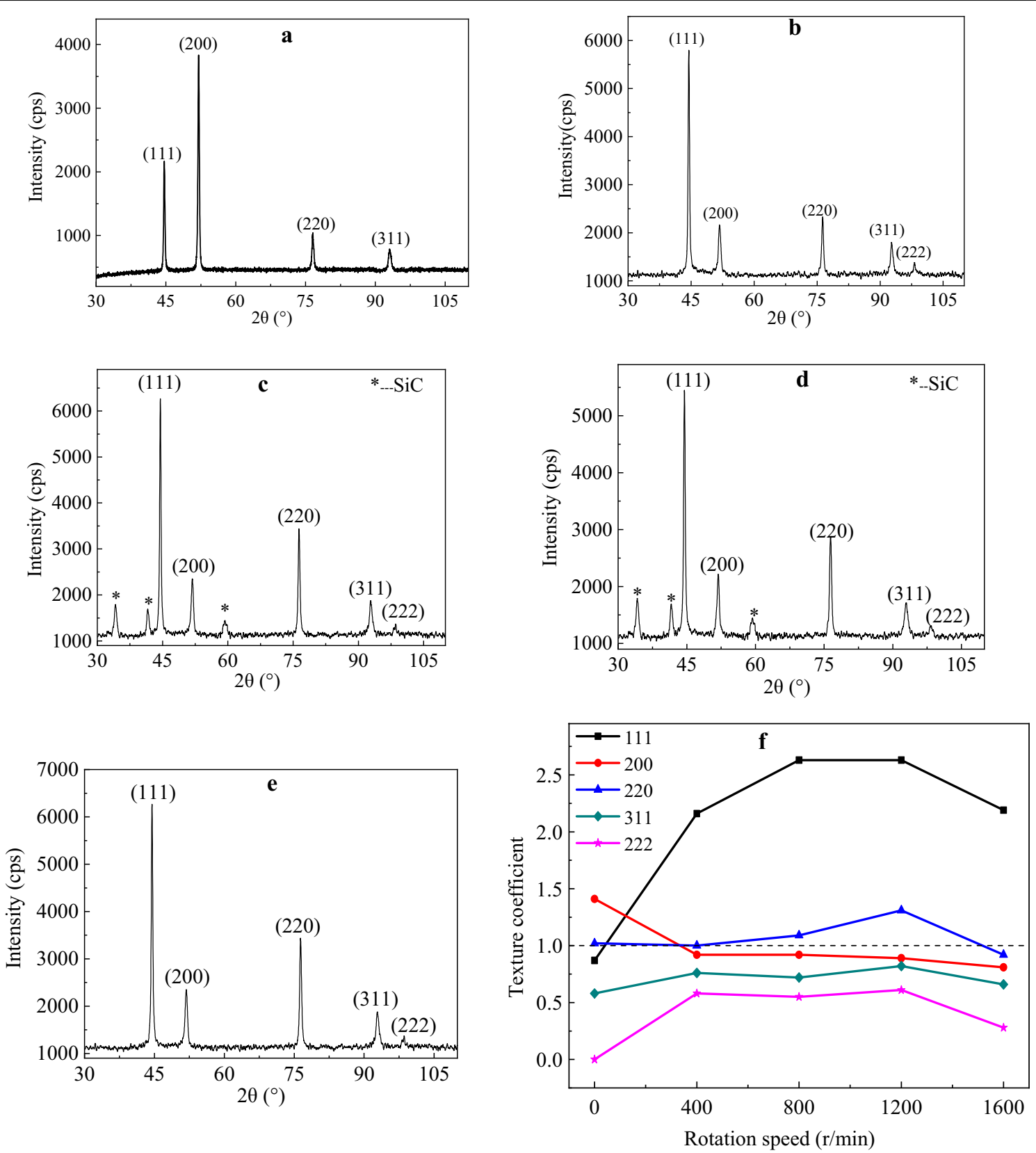

Figure 4 XRD patterns of deposited Ni-Co/SiC coatings under rotation speeds of: a 0, b $400 \mathrm{r} / \mathrm{min}, \mathbf{c} 800 \mathrm{r} / \mathrm{min}$, d $1200 \mathrm{r} / \mathrm{min}$ and e $1600 \mathrm{r} / \mathrm{min}$. f Texture coefficient versus rotation coefficient

\subsection{Microhardness of the Coating}

The relationship between the microhardness of the obtained $\mathrm{Ni}-\mathrm{Co} / \mathrm{SiC}$ coatings and the rotation speed is shown in Figure 6. As can be seen, the microhardness values achieved under the supergravity field were higher than that at normal gravity. For rotation speeds no greater than $800 \mathrm{r} / \mathrm{min}$, the microhardness values increased sharply and reached the maximum value of $680 \mathrm{HV}$ at $800 \mathrm{r} / \mathrm{min}$. Thereafter, the microhardness values decreased for rotation speeds higher than $800 \mathrm{r} /$ min, becoming about $510 \mathrm{HV}$. As mentioned above, the dispersion of $\mathrm{SiC}$ nanoparticles into the coatings could decrease the grain size. Based on the Hall-Petch relationship [35], the internal dislocation movement determines the degree of deformation of the coatings, and the grain boundary can effectively hinder the dislocation movement. Smaller grains produce a greater level of grain boundaries and consequently provide superior 


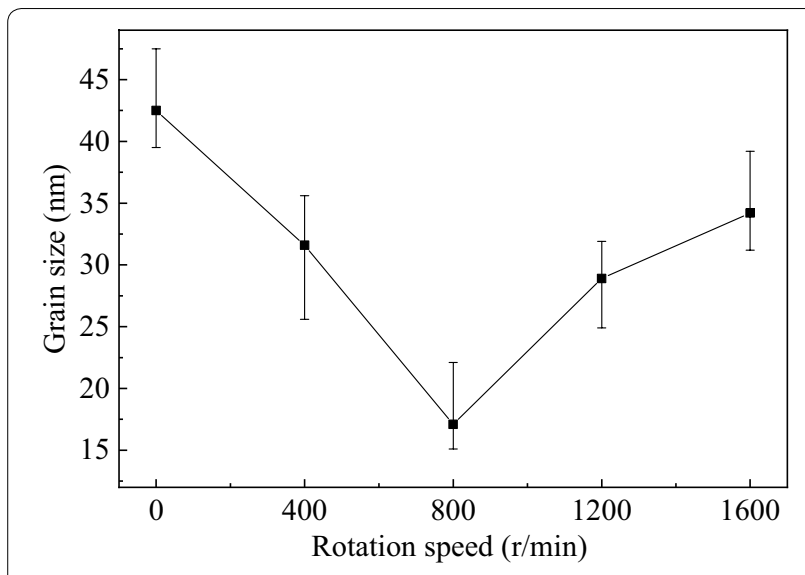

Figure $\mathbf{5}$ Grain size as a function of rotation speed

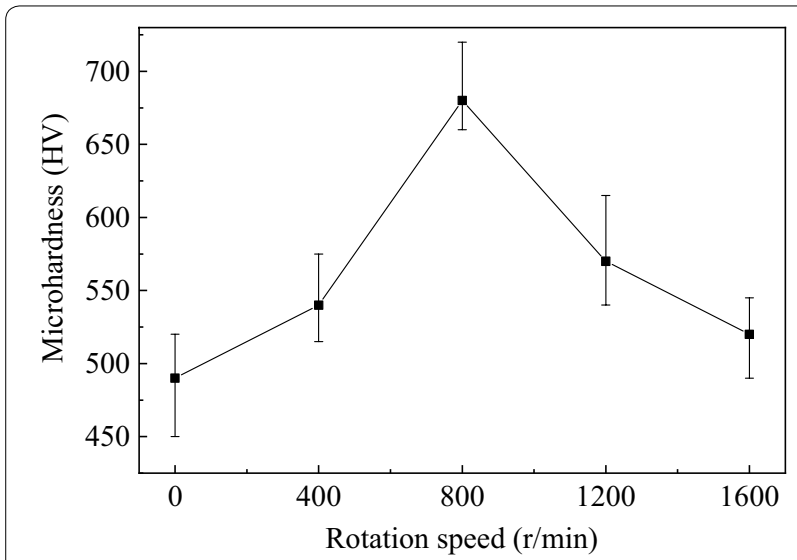

Figure 6 Microhardness values for the $\mathrm{Ni}-\mathrm{Co} / \mathrm{SiC}$ coatings prepared at: a 0, b $400 \mathrm{r} / \mathrm{min}, \mathbf{c} 800 \mathrm{r} / \mathrm{min}$, d $1200 \mathrm{r} / \mathrm{min}$, and e $1600 \mathrm{r} / \mathrm{min}$

impediment to dislocation movement, leading to reinforcement of the microhardness. In addition, due to the addition of $\mathrm{SiC}$ into the matrix, plastic deformation of the
Ni-Co matrix would result in a shrinking effect because of the dispersion-strengthening effect, resulting in a dramatic reinforcement of coating microhardness [36]. However, a rotation speed greater than $800 \mathrm{r} / \mathrm{min}$ would contribute to the formation of larger grain sizes, thus lowering the coating microhardness. However, such high speeds would also contribute to a decreasing $\mathrm{SiC}$ content in the coating, as discussed above, which, correspondingly, would reduce the dispersion-strengthening effect.

\subsection{Wear Resistance}

The data for the wear resistance tests are presented in Figure 7. It can be seen that the friction coefficient obtained with a normal gravity field is much larger than the values achieved under the supergravity field, as shown in Figure 7a. As the rotation speed is increased to $800 \mathrm{r} /$ min, the friction coefficient decreased and then increases moderately when the rotation speed exceeds $800 \mathrm{r} / \mathrm{min}$. The minimal value for the friction coefficient is approximately 0.13 . It can also be observed that the wear weight change shows a similar trend as illustrated in Figure $7 \mathrm{~b}$. The smallest wear weight loss obtained is about $1.4 \mathrm{mg}$. The SEM images for the worn surfaces of the as-prepared coatings after the wear tests are presented in Figure 8. It is evident that the prepared $\mathrm{Ni}-\mathrm{Co} / \mathrm{SiC}$ coatings sustained adhesive wear. Generally, the coatings deposited under the supergravity field conditions are less worn than those for the normal gravity field. Although ploughing is evident on all worn coatings, much more spalling are generated on the coatings formed under the normal gravity field, as shown in Figure 8a. Less spalling can be seen on the worn surfaces with a rotation speed of $400 \mathrm{r} / \mathrm{min}$ as shown in Figure $8 \mathrm{~b}$. No visible spalling can be observed as the rotation speed is varied from $800 \mathrm{r} / \mathrm{min}$ to $1200 \mathrm{r} /$ min. A relatively small level of spalling starts to appear on the worn surface obtained at $1600 \mathrm{r} / \mathrm{min}$, as depicted in Figure 8e. Moreover, the spalling trace generated on the
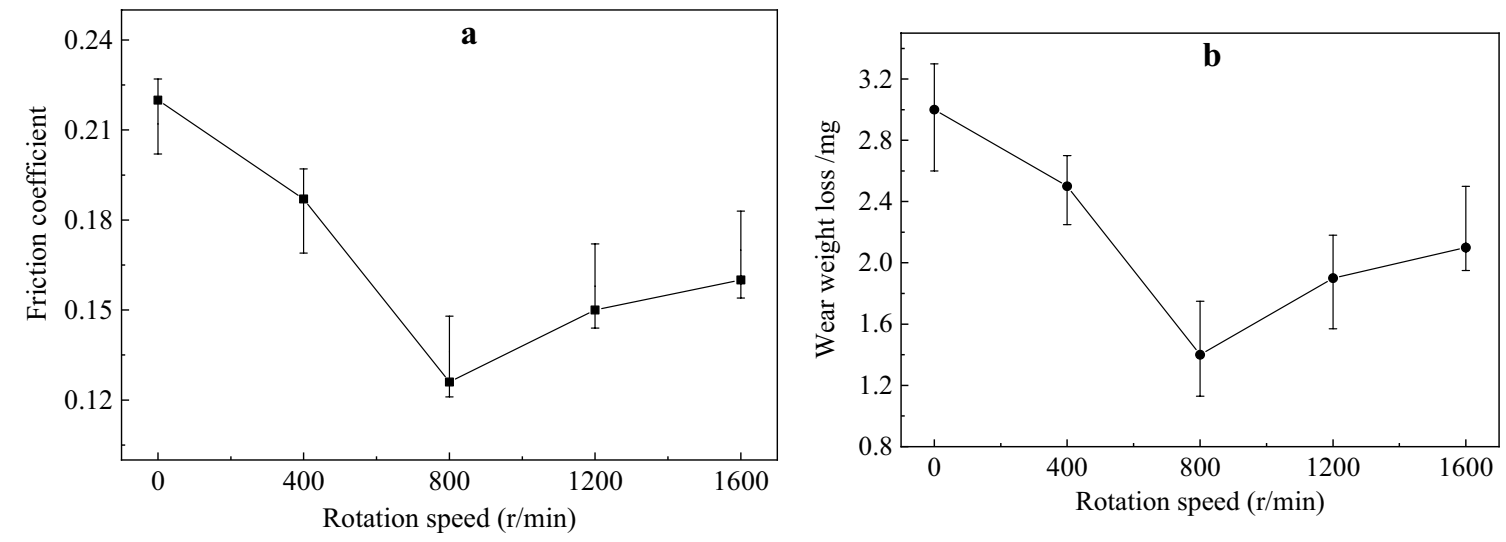

Figure 7 Effect of the supergravity field on: $\mathbf{a}$ the friction coefficient, and $\mathbf{b}$ the wear weight loss 

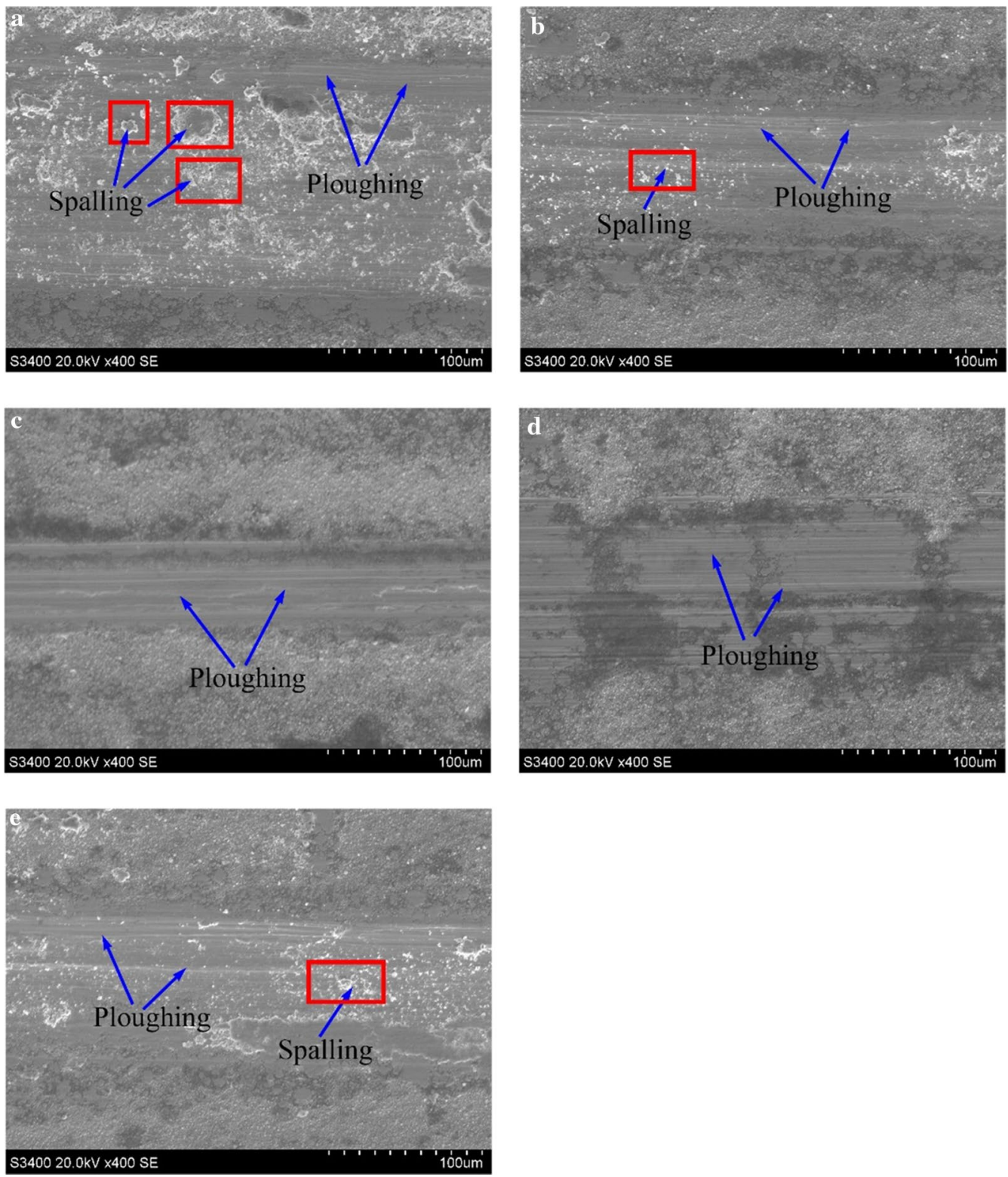

Figure 8 SEM images of the worn surfaces of $\mathrm{Ni}-\mathrm{Co} / \mathrm{SiC}$ coatings obtained at different rotation speeds: a 0, b $400 \mathrm{r} / \mathrm{min}, \mathbf{c} 800 \mathrm{r} / \mathrm{min}, \mathbf{d} 1200 \mathrm{r} / \mathrm{min}$ and $\mathbf{e} 1600 \mathrm{r} / \mathrm{min}$

surface corresponding to $800 \mathrm{r} / \mathrm{min}$ is the smallest among the coatings.

Based on the Archard model [37], hardness has positive effects on wear resistance to some extent. It is intuitive that the higher the microhardness value is for a material the better will be the wear resistance and consequently the smaller will be the friction coefficient and the wear weight loss. Also, the surface morphology makes a contribution to wear resistance. That is, a smoother surface morphology is beneficial to achieving a better wear resistance [38].

\section{Conclusions}

(1) In comparison with electrodeposition under normal gravity, supergravity field-enhanced electrodeposition resulted in a smoother $\mathrm{Ni}-\mathrm{Co} / \mathrm{SiC}$ coating surface morphology, which was partly due to the uniform dispersion of $\mathrm{SiC}$ nanoparticles in the coatings under the enhanced gravity conditions. The more nucleation sites offered by the $\mathrm{SiC}$ nanoparticle also contributed to the realization of a flat surface. 
(2) In general, the $\mathrm{SiC}$ content of the $\mathrm{Ni}-\mathrm{Co} / \mathrm{SiC}$ coatings prepared with a supergravity enhanced field was higher than that achieved for conventional electrodeposition. For a specific range of rotation speeds, the $\mathrm{SiC}$ content increased monotonically to a maximum value. Further increases in rotation speed, however, led to reductions in the $\mathrm{SiC}$ content of the coatings. The highest $\mathrm{SiC}$ content achieved was $8.1 \mathrm{wt} \%$.

(3) The distribution of $\mathrm{SiC}$ nanoparticles in the $\mathrm{Ni}-\mathrm{Co} /$ $\mathrm{SiC}$ coatings constrained dislocation movement and hence the strength and microhardness of the coatings increased. The largest microhardness value achieved under the supergravity field conditions was $680 \mathrm{HV}$, indicating a remarkable enhancement of microhardness in contrast to that obtained with conventional electrodeposition.

(4) The $\mathrm{Ni}-\mathrm{Co} / \mathrm{SiC}$ coatings produced by supergravity field-enhanced electrodeposition showed noticeably improved wear resistance as compared to conventional electrodeposition. At $800 \mathrm{r} / \mathrm{min}$, the $\mathrm{Ni}-\mathrm{Co} / \mathrm{SiC}$ coating exhibited the lowest wear weight loss of $1.4 \mathrm{mg}$ as well as the smallest average friction coefficient of 0.13 . These conditions also yielded the narrowest wear scar due to grain refinement and the dispersion-strengthening effect of the $\mathrm{SiC}$ nanoparticles.

\section{Acknowledgements}

The authors sincerely thanks to the team members in our research group for their critical discussion and reading during manuscript preparation.

\section{Authors' Contributions}

$X Y$ was in charge of the whole trial and wrote the manuscript; NS assisted with sampling and laboratory analyses. All authors read and approved the final manuscript.

\section{Funding}

Supported by National Key Research and Development Program of China (Grant No. 2018YFB1 105900), National Basic Research Program of China (973 Program, Grant No. 2015CB057502), and Fundamental Research Funds for the Central Universities (Grant No. NZ2016106).

\section{Competing Interests}

The authors declare that they have no competing interests.

\section{Author Details}

${ }^{1}$ Nanjing University of Aeronautics and Astronautics, Nanjing 210016, China. 2 Jiangsu Key Laboratory of Precision and Micro-Manufacturing Technology, Nanjing 210016, China.

Received: 26 December 2019 Revised: 17 July 2020 Accepted: 13 August 2020

Published online: 27 August 2020

\section{References}

[1] K Ignatova, Y Marcheva. Composition and structure of Ni-Co coating depending on the ratio of $\mathrm{Ni}$ and $\mathrm{Co}$ in a citrate electrolyte. Bulgarian Chemical Communications, 2017, 49(2): 313-319.

[2] A Sivanantham, P Ganesan, S Shanmugam. Hierarchical $\mathrm{NiCO}_{2} \mathrm{~S}_{4}$ nanowire arrays supported on $\mathrm{Ni}$ foam: an efficient and durable bifunctional electrocatalyst for oxygen and hydrogen evolution reactions. Advanced Functional Materials, 2016, 26(26): 4661-4672.

[3] C Du, L Yang, F Yang, et al. Nest-like NiCoP for highly efficient overall water splitting. ACS Catalysis, 2017, 7(6): 4131-4137.

[4] J De, T Banerjee, R S Sen, et al. Multi-objective optimization of electroless ternary Nickel-Cobalt-Phosphorous coating using non-dominant sorting genetic algorithm-II. Engineering Science and Technology, An International Journal, 2016, 19(3): 1526-1533.

[5] A Karimzadeh, M Aliofkhazraei, F C Walsh. A review of electrodeposited $\mathrm{Ni}-\mathrm{Co}$ alloy and composite coatings: Microstructure, properties and applications. Surface and Coatings Technology, 2019, 372: 463-498.

[6] Y J Chang, C H Chang. Intelligent computation for optimal fabrication condition of a protein chip with $\mathrm{Ni}$-Co alloy-coated surface. Journal of Laboratory Automation, 2016, 21(3): 394-401.

[7] S M L Baghal, A Amadeh, M H Sohi, et al. The effect of SDS surfactant on tensile properties of electrodeposited $\mathrm{Ni}-\mathrm{Co} / \mathrm{SiC}$ nanocomposites. Materials Science and Engineering A-Structural Materials Properties Microstructure and Processing, 2013, 559: 583-590.

[8] C Ma, S Wang, F C Walsh. The electrodeposition of nanocrystalline CobaltNickel-Phosphorus alloy coatings: A review. Transactions of the IMF, 2015, 93(5): 275-280.

[9] B Bakhit, A Akbari. A comparative study of the effects of saccharin and $\beta$-SiC nano-particles on the properties of $\mathrm{Ni}$ and $\mathrm{Ni}-\mathrm{Co}$ alloy coatings. Surface and Coatings Technology, 2014, 253: 76-82.

[10] A Rasooli, M S Safavi, M K Hokmabad. $\mathrm{Cr}_{2} \mathrm{O}_{3}$ nanoparticles: A promising candidate to improve the mechanical properties and corrosion resistance of $\mathrm{Ni}-\mathrm{Co}$ alloy coatings. Ceramics International, 2018, 44(6): 6466-6473.

[11] A Al-Fatesh. Suppression of carbon formation in $\mathrm{CH}_{4}-\mathrm{CO}_{2}$ reforming by addition of $\mathrm{Sr}$ into bimetallic $\mathrm{Ni}-\mathrm{Co} / \mathrm{Y}-\mathrm{Al}_{2} \mathrm{O}_{3}$ catalyst. Journal of King Saud University - Engineering Sciences, 2015, 27(1): 101-107.

[12] X H Wang, B Lu, Z F Hu, et al. Effects of $n-\mathrm{Al}_{2} \mathrm{O}_{3}$ particles content on structure and performance of electro-brush plating $\mathrm{Ni}$-Co alloy composite coatings. Rare Metal Materials and Engineering, 2016, 45(1): 36-41.

[13] A W J Hsue, Y F Chang. Toward synchronous hybrid micro-EDM grinding of micro-holes using helical taper tools formed by $\mathrm{Ni}$-Co/diamond $\mathrm{Co}-$ deposition. Journal of Materials Processing Technology, 2016, 234: 368-382.

[14] P R Dheeraj, A Patra, S Sengupta, et al. Synergistic effect of peak current density and nature of surfactant on microstructure, mechanical and electrochemical properties of pulsed electrodeposited Ni-Co-SiC nanocomposites. Journal of Alloys and Compounds, 2017, 729: 1093-1107.

[15] B Bakhit. The influence of electrolyte composition on the properties of $\mathrm{Ni}-\mathrm{Co}$ alloy coatings reinforced by SiC nano-particles. Surface and Coatings Technology, 2015, 275: 324-331.

[16] CY Ma, D Q Zhao, Z P Ma. Effects of duty cycle and pulse frequency on microstructures and properties of electrodeposited Ni-Co-SiC nanocoatings. Ceramics International, 2012, 46(8): 12128-12137.

[17] W Jiang, L D Shen, M Y Xu, et al. Mechanical properties and corrosion resistance of $\mathrm{Ni}-\mathrm{Co}-\mathrm{SiC}$ composite coatings by magnetic field-induced jet electrodeposition. Journal of Alloys and Compounds, 2019, 791: 847-855.

[18] B Bakhit, A Akbari. Effect of particle size and co-deposition technique on hardness and corrosion properties of $\mathrm{Ni}-\mathrm{Co} / \mathrm{SiC}$ composite coatings. Surface and Coatings Technology, 2012, 206(23): 4964-4975.

[19] H K Lee, HY Lee, J M Jeon. Codeposition of micro- and nano-sized SiC particles in the nickel matrix composite coatings obtained by electroplating. Surface and Coatings Technology, 2007, 201 (8): 4711-4717.

[20] W Wang, FY Hou, H Wang, et al. Fabrication and characterization of $\mathrm{Ni}-\mathrm{ZrO}_{2}$ composite nano-coatings by pulse electrodeposition. Scripta Materialia, 2005, 53(5): 613-618.

[21] N Zhang, Z Wang, L Guo, et al. Rapid fabrication of W-Cu composites via low-temperature infiltration in supergravity fields. Journal of Alloys and Compounds, 2019, 809: 151782.

[22] N Zhang, Z Wang, L Guo, et al. Supergravity process for enriching and separating Ag from $\mathrm{Sn}-\mathrm{Ag}-\mathrm{Zn}$ melts. Chemical Engineering and Processing - Process Intensification, 2019, 143: 107591. 
[23] X C Yin, G Sun, A L Song, et al. A novel structure of Ni-(MoS $2 / G O)$ composite coatings deposited on Ni foam under supergravity field as efficient hydrogen evolution reaction catalysts in alkaline solution. Electrochimica Acta, 2017, 249: 52-63.

[24] A Murotani, T Fuchigami, M Atobe. Electrochemical deposition of Ni/SiC under centrifugal fields. Electrochemistry, 2008, 76(11): 824-826.

[25] X Y Hu, N S Qu. Improved corrosion resistance of Ni-Co coatings prepared by electrodeposition with large centrifugal acceleration. Journal of Materials Engineering and Performance, 2019, 28(4): 2104-2114.

[26] XY Hu, N S Qu. Enhanced corrosion resistance of nickel-cobalt/carborundum coatings formed by supergravity field-assisted electrodeposition. Thin Solid Films, 2020, 700: 137923

[27] M Srivastava, V K W Grips, K S Rajam. Electrodeposition of Ni-Co composites containing nano- $\mathrm{CeO}_{2}$ and their structure, properties. Applied Surface Science, 2010, 257(3): 717-722.

[28] J S Chen, Y H Huang, Z D Liu. Jet electrodeposition oriented by rapid prototyping. Transactions of Nonferrous Metals Society of China, 2005, 15(3): 247-250.

[29] W Jiang, L D Shen, M B Qiu, et al. Preparation of Ni-SiC composite coatings by magnetic field-enhanced jet electrodeposition. Journal of Alloys and Compounds, 2018, 762: 115-124.

[30] N Guglielmi. Kinetics of the deposition of inert particles from electrolytic baths. Journal of the Electrochemical Society, 1972, 119(8): 1009.

[31] D D Ning, A Zhang, M Murtaza, et al. Effect of surfactants on the electrodeposition of $\mathrm{Cu}-\mathrm{TiO}_{2}$ composite coatings prepared by jet electrodeposition. Journal of Alloys and Compounds, 2019, 777: 1245-1250.

[32] F T L Muniz, M A Miranda, C M Santos, et al. The Scherrer equation and the dynamical theory of X-ray diffraction. Acta Cryst., 2016, 72(3): 385-390.

[33] L Benea, P L Bonora, A Borello, et al. Preparation and investigation of nanostructured SiC-nickel layers by electrodeposition. Solid State lonics, 2002, 151(1-4): 89-95.

[34] N P Wasekar, L Bathini, G Sundararajan. Tribological behavior of pulsed electrodeposited Ni-W/SiC nanocomposites. Journal of Materials Engineering and Performance, 2018, 27(10): 5236-5245.

[35] J Hu, Y N Shi, X Sauvage, et al. Grain boundary stability governs hardening and softening in extremely fine nanograined metals. Science, 2017, 355 (6331): 1292-1296

[36] Y J Xue, W Ma, J S Li, et al. Fabrication and wear resistance of $\mathrm{Ni}-\mathrm{CeO}_{2}$ nanocomposite coatings by electrodeposition under ultrasound condition. Advanced Tribology, 2010, 3: 202-313.

[37] A A P Sidharth, I Sridhar. Material removal analysis for compliant polishing tool using adaptive meshing technique and Archard wear model. Wear, 2019, 418: 140-150.

[38] S Dehgahi, R Amini, M Alizadeh. Corrosion, passivation and wear behaviors of electrodeposited $\mathrm{Ni}-\mathrm{Al}_{2} \mathrm{O}_{3}-\mathrm{SiC}$ nanocomposite coatings. Surface and Coatings Technology, 2016, 304: 502-511.

\section{Submit your manuscript to a SpringerOpen ${ }^{\circ}$ journal and benefit from:}

- Convenient online submission

- Rigorous peer review

- Open access: articles freely available online

- High visibility within the field

- Retaining the copyright to your article

Submit your next manuscript at $\boldsymbol{\nabla}$ springeropen.com 